\title{
Simulation Evaluation of Fast Frequency Response Capacity of New Energy Power Stations
}

\author{
Chang Wanzheng ${ }^{1, *}$, Wang Nan ${ }^{1}$, Cheng Yan $^{1}$, Sun Shumin ${ }^{1}$, Wang Yanzhuo ${ }^{1}$, Li Qinghua ${ }^{1}$, Zhang Zhihao ${ }^{1}$ \\ ${ }^{1}$ State Grid Shandong Electric Power Company Electric Power Research Institute, Jinan, Shandong, 250002, China
}

\begin{abstract}
The number of new energy power stations in China is boosting in recent years, a large number of conventional power supplies have been replaced, resulting in severe problems in controlling the frequency of power grids and easily leading to frequency issues of the state power grid. When large frequency disturbance occurs in power grid, renewable energy stations can participate in primary frequency regulation of power grid and provide support for system frequency, and the primary frequency regulation capability of the large power grid in sending end is significantly enhanced. In this study, a simulation evaluation method was proposed for the FFR controller of new energy power stations based on the RT-LAB real-time simulation analysis platform. The FFR controller was connected to in the simulation model of the state power grid via I/O physical interfaces and MODBUS communication, so as to conduct the step disturbance test, anti-disturbance performance verification test and automatic generation control (AGC) coordination test and hardware-in-theloop simulation test of the controller. Results of the tests proved that the test method proposed in this study is accurate and reliable, and help improve the performance of a new energy power station in the state power grid.
\end{abstract}

\section{Introduction}

To cope with the shortage of fossil energy and in the face of the environmental pollution it brings about, power generation using renewable energies, represented by wind power generation and photovoltaic power generation, has been attracting wide attention for its low carbon consumption, environmental-friendliness, proven technology and capability of large-scale production ${ }^{[1]}$. The number of new energy power stations in China is boosting in recent years, but as new energy power generation requires a huge number of electronic power transform devices and connection to the state power grid and a large number of conventional power supplies have been replaced, the new energy power generation system is of a low inertia level. Besides, its reserve capacity decreases and the rotational inertia and capability of frequency regulation keep decreasing. AC and DC failure also causes significant power deficiency, resulting in severe problems in controlling the frequency of power grids and easily leading to frequency issues of the state power grid ${ }^{[2]}$. Given the low-frequency response of new energy power stations and great stress and high cost to regulate the frequency and peak of conventional power units, primary frequency regulation capacity of new energy power stations is the development trend of the new energy power generation technology. It has been expressly stated in GB 38755-2019 Code on Security and Stability for Power System, implemented on July 1, 2021, that "wind power stations and photovoltaic generation

\footnotetext{
* Chang Wanzheng: 15763560195@163.com
}

stations shall be provided with primary frequency regulation".

There still lack domestic and foreign standards on the fast frequency response (FFR) test for new energy power stations to be connected to the state power grid, so it is urgent to conduct both theoretical research and practical tests on FFR capacity of new energy power stations and to decide the method to test whether a new energy power unit is capable of primary frequency regulation and can be connected to a power grid.

According to literature, reactive power regulating equipment in wind power stations had been tested for voltage regulation capacity, reactive power regulation capacity and bus power quality [3]; inverter-based photovoltaic power stations had been tested for the performance of the reactive voltage control system, range of reactive power regulation and dynamic response of inverters but new energy power stations in power grids were not tested for the FFR capacity ${ }^{[4]}$; new energy power stations in the sending state grid had been measured and analyzed for FFR to test the primary frequency regulation capacity of the sending state grid in Northwest China that involved a large number of new energy power stations, the result of which showed that photovoltaic power units and wind power units had better FFR than conventional ones and better helped improve the safety and stability of the frequency of the sending state grid, but no research and simulation tests had been conducted for the FFR capacity of new energy power units based on the semi-physical simulation platform ${ }^{[5]}$; wind power units and photovoltaic inverters had been performed semi-physical simulation 
low-voltage traverse tests based on the semi-physical simulation platform, but wind power stations and photovoltaic power stations had not been tested for the FFR capacity based on this platform ${ }^{[6][7]}$.

In this study, a hardware-in-the-loop simulation test method was proposed for the FFR controller of new energy power stations based on the RT-LAB real-time simulation analysis platform. The FFR controller was connected to in the simulation model of the state power grid via $\mathrm{I} / \mathrm{O}$ physical interfaces and MODBUS communication, so as to conduct the step disturbance test, anti-disturbance performance verification test and automatic generation control (AGC) coordination test and hardware-in-the-loop simulation test of the controller. Results of the tests proved that the test method proposed in this study is accurate and reliable, and could test and verify the performance of a new energy power unit connected to the state power grid, provide experimental data for optimizing the operation strategy of such a unit and help improve the performance of a new energy power station in the state power grid.

\section{Building of the RT-LAB semi-physical simulation platform}

\subsection{RT-LAB real-time simulation platform}

In terms of the application of electronic power technology, RT-LAB is a digital dynamic-model real-time simulation platform enabling software and hardware extensions. It is highly efficient in directly applying mathematical models of dynamic system built in MATLAB/Simulink to realtime simulation tests and can truly connect hardware to the simulation test loop.

In this study, the semi-physical simulation test program to connect the FFR controller of the new energy power station to the mathematical model of dynamic system built in MATLAB/Simulink was propose to test the controller under the required overall performance of the system, so as to effectively improve the reliability of simulation tests.

\subsection{Hardware connection}

Hardware of the built RT-LAB semi-physical real-time simulation system included the FFR controller of the new energy power station and the RT-LAB simulation host and simulation target, and its software included MATLAB/Simulink, the underlying program of RT-LAB, the ARTEMIS toolbox and the RT-Events toolbox. The composition of the test system is as shown in Fig.1. A simulation model of a wind power station with a centralized grid connection was built, in which the FFR controller of this new energy power station was connected to the RT-LAB platform via I/O physical interfaces and MODBUS communication. This model and the simulation model of the power grid were both built in MATLAB/Simulink.

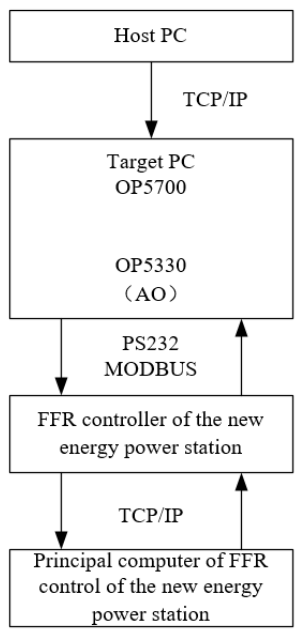

Fig.1 Structure of the test system

\subsection{Simulation model}

When building a simulation model in Simulink, the toplevel subsystems were encapsulated and named as SM_, SC_ and SS_with the prefix names. The SM_ subsystem was responsible for real-time computation and network synchronization of the model. A system must and could contain only one SM_ subsystem. When the system ran distributed computing with multiple cores, the model must also contain the $\mathrm{SS}_{-}$subsystem that contained the computing unit of the model. The SC subsystem contained the basic modules to collect and display data, and was responsible for the real-time monitoring of key parameters and curves of the simulation system, as well as post-computing of data communication between subsystems.

There were three subsystems in the FFR system of the new energy power station. The main system SM_system mainly simulated the topology of the main circuit, including the state grid model, the $110 \mathrm{kV}$ line, the 110 $\mathrm{kV} / 35 \mathrm{kV}$ boost transformer, the grid connection switch of the wind power station and the models inside the station. The subsystem SS_ctrol mainly stimulated the FFR controller of the station and the models of the $\mathrm{I} / \mathrm{O}$ interfaces and MODBUS communication of RT-LAB. The subsystem SC_console mainly displayed the waveforms, the signature voltage and current of the station, the active commands of the controller, the active command of AGC, the theoretical power, the actual power and frequency. The overall Structure of the model is as shown below.

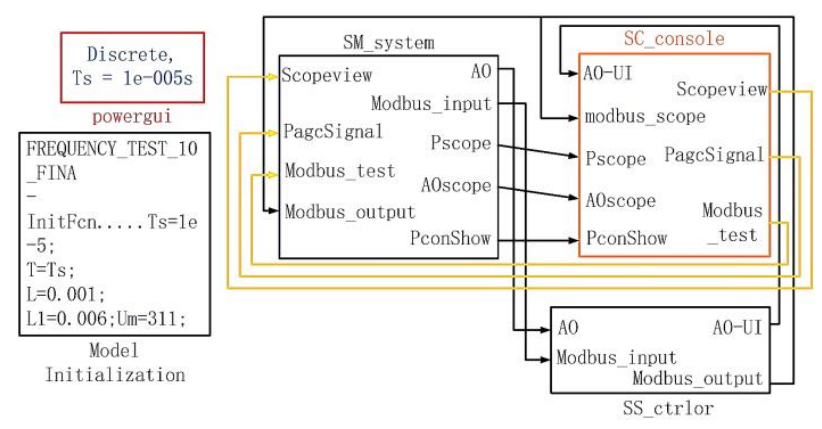

Fig.2 Overall structure of the model 
The FFR controller of the station was connected to the RT-LAB simulation model via $\mathrm{I} / \mathrm{O}$ interfaces and MODBUS-TCP communication. There were four types of such I/O interfaces, namely the analog output (AO) interface, the analog input (AI) interface, the digital output (DO) interface and the digital input (DI) interface. AO and DO referred to the analog and 0-1 digital quantity of the judge mark output by the model and collected by the hardware controller, and AI and DI referred to the analog and 0-1 digital quantity of the judge mark output by the hardware controller and collected by the model. In this model, AO communication was used to transport analog measurements, including the measured three-phase AC voltage, three-phase AC current and ground signal at the grid connection point of the station, from the power grid to the FFR controller of the station.

The MODBUS-TCP communication model contained the remote signal value, remote measurement value, remote modulation value and remote control value, of which the remote signal value and remote measurement value were the $0-1$ variable and analog of the hardware controller collected by the Simulink model and the remote modulation value and remote control value were the $0-1$ variable and analog of the Simulink model collected by the hardware controller. The model built in this study also contained the same values, of which the remote measurement value referred to the command value, i.e., the active command value of the controller, input by the FFR controller to the simulation test model via the MODBUS-TCP module, the remote signal value referred to the 0-1 digital quantity, including the grid connection switching quantity and command input state quantity of the controller, input by the FFR controller to the simulation test model via the MODBUS-TCP module, the remote control value referred to the command value, i.e., the active command value of AGC, input by the simulation test model to the FFR controller via the MODBUS-TCP module, and the remote modulation value referred to the 0-1 digital quantity, i.e., the input state quantity of AGC, input by the simulation test model to the FFR controller via the MODBUS-TCP module.

\section{Example}

\subsection{Test content}

A 49.5 MW wind power station, which was connected to the power grid via a $110 \mathrm{kV}$ bus, was simulated in this study. Each simulation test was conducted under the corresponding working condition, which is detailed as follows:

1) Working condition 1: When the wind power station was subject to limited power and the output range was $20 \%$ to $30 \%$ of the rated capacity of the station;

2) Working condition 2: When the wind power station was not subject to limited power and the output range was $20 \%$ to $30 \%$ of the rated capacity of the station;

3) Working condition 3: When the wind power station was subject to limited power and the output range was $50 \%$ to $90 \%$ of the rated capacity of the station;
4) Working condition 4: When the wind power station was not subject to limited power and the output range was $50 \%$ to $90 \%$ of the rated capacity of the station.

Three simulation tests were conducted in this study:

(1) Frequency step disturbance test: The step disturbance was divided into step up disturbance and step down disturbance. The former referred to the process where the frequency stepped from $50 \mathrm{~Hz}$ to $50.20 \mathrm{~Hz}$, stayed at $50.20 \mathrm{~Hz}$ for 20 seconds and restored to $50 \mathrm{~Hz}$, and the latter referred to the process where the frequency stepped from $50 \mathrm{~Hz}$ to $49.80 \mathrm{~Hz}$, stayed at $49.80 \mathrm{~Hz}$ for 20 seconds and restored to $50 \mathrm{~Hz}$. This test, simulating the primary frequency regulation test for conventional hydraulic power stations and thermal power stations to be connected to the power grid, was repeated 6 times, including four times to test the frequency step up disturbance under working conditions 1, 2, 3 and 4, respectively, and two times to test the frequency step up disturbance under working conditions 1 and 3 , respectively, intending to test the response of the wind power station under frequency step disturbance. The FFR capacity was then evaluated according to the analysis of the response time and reactive power before and after step disturbance.

(2) Anti-disturbance performance verification test: This test was conducted under the working condition 1 . The frequency signal generator was used to simulate the transient process, such as traversing of high and low voltage in the power grid, and output the following two verification signals to check whether the FFR of the station malfunctioned. Signal 1: A certain phase of the computing frequency of the FFR control system was selected, the voltage amplitude was made instantly fall to $(0 \%, 20 \%$, $40 \%, 60 \%$ and $80 \%$ ) of the rated voltage for $150 \mathrm{~ms}$, and two phase shifts of $60^{\circ}$ were made when the voltage fell and restored. Signal 2: Three phases were selected, the voltage amplitude was made instantly step to $(115 \%$, $120 \%, 125 \%$ or $130 \%$ ) of the rated voltage for $500 \mathrm{~ms}$, and two phase shifts of $60^{\circ}$ were made when the voltage stepped and restored.

(3) AGC coordination test: This test was to verify whether the FFR capacity of the station could well coordinate with the secondary frequency regulation command of the dispatching end. Both of the two commands were executed under the working condition 3 , that is, the second command was sent when the first command had been responded to and was being executed. This test was repeated 8 times under frequency disturbance and with increased or decreased output.

1) Frequency stepping from $50 \mathrm{~Hz}$ to $50.20 \mathrm{~Hz}+10 \%$ increase in the rated capacity of the station at secondary frequency regulation ("+" indicates that the latter was sent and executed when the former had been responded to and was being executed);

2) $10 \%$ increase in the rated capacity of the station at secondary frequency regulation + frequency stepping from $50 \mathrm{~Hz}$ to $50.20 \mathrm{~Hz}$;

3) Frequency stepping from $50 \mathrm{~Hz}$ to $50.20 \mathrm{~Hz}+10 \%$ decrease in the rated capacity of the station at secondary frequency regulation; 4) $10 \%$ decrease in the rated 
capacity of the station at secondary frequency regulation + frequency stepping from $50 \mathrm{~Hz}$ to $50.20 \mathrm{~Hz}$;

5) Frequency stepping from $50 \mathrm{~Hz}$ to $49.80 \mathrm{~Hz}+10 \%$ increase in the rated capacity of the station at secondary frequency regulation;

6) $10 \%$ increase in the rated capacity of the station at secondary frequency regulation + frequency stepping from $50 \mathrm{~Hz}$ to $49.80 \mathrm{~Hz}$;

7) Frequency stepping from $50 \mathrm{~Hz}$ to $49.80 \mathrm{~Hz}+10 \%$ decrease in the rated capacity of the station at secondary frequency regulation;

8) $10 \%$ decrease in the rated capacity of the station at secondary frequency regulation + frequency stepping from $50 \mathrm{~Hz}$ to $49.80 \mathrm{~Hz}$;

\subsection{Data analysis}

\subsubsection{Result of the frequency step disturbance test}

Scopeview waveform analysis of RT-LAB was used to analyze the frequency step disturbance test. Fig.3 and Fig.4 are the waveform diagram and process analysis diagram of the wind power station when the frequency stepped to $50.20 \mathrm{~Hz}$ under the working condition 1 . The wave was recorded for 30 seconds in the test, during which the response time was 0.522 seconds, the active power before step disturbance was $12.00 \mathrm{MW}$ and that after step disturbance was $7.05 \mathrm{MW}$, all meeting the requirement.
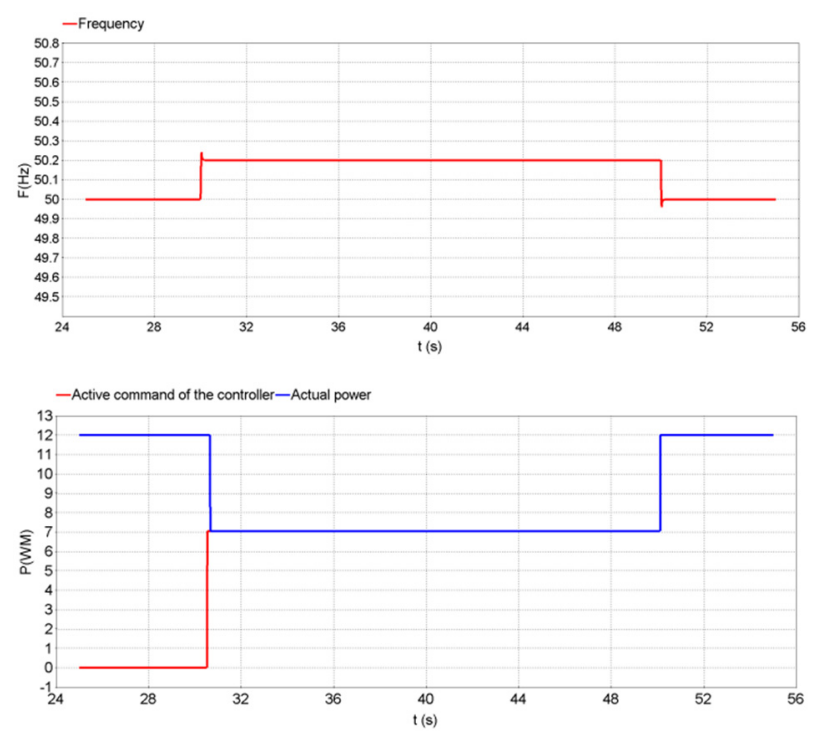

Fig.3 Response when the frequency stepped from $50 \mathrm{~Hz}$ to $50.20 \mathrm{~Hz}$ for 20 seconds and the active command of AGC was $12 \mathrm{MW}$ at limited power under the working condition 1

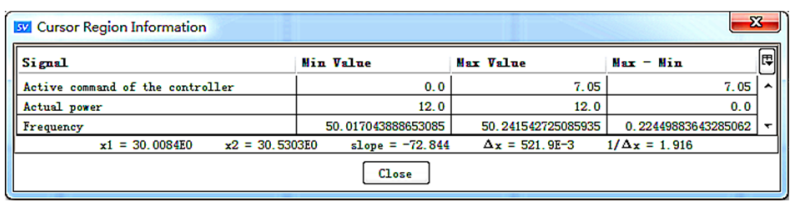

Fig.4 Analysis of the process where the frequency stepped from $50 \mathrm{~Hz}$ to $50.20 \mathrm{~Hz}$ under the working condition 1

\subsubsection{Result of the anti-disturbance performance verification test}

Scopeview waveform analysis of RT-LAB was used to analyze the anti-disturbance performance verification test. Fig. 5 is the waveform diagram when the phase-A voltage amplitude of the wind power station instantly fell to $20 \%$ and two phase shifts of $60^{\circ}$ were made when the voltage fell and restored. The figure shows that during the low voltage traversing in the stimulated power grid, the active command of the FFR controller of the station did not act, that is, the FFR capacity of the station did not act reliably, meeting the requirement.
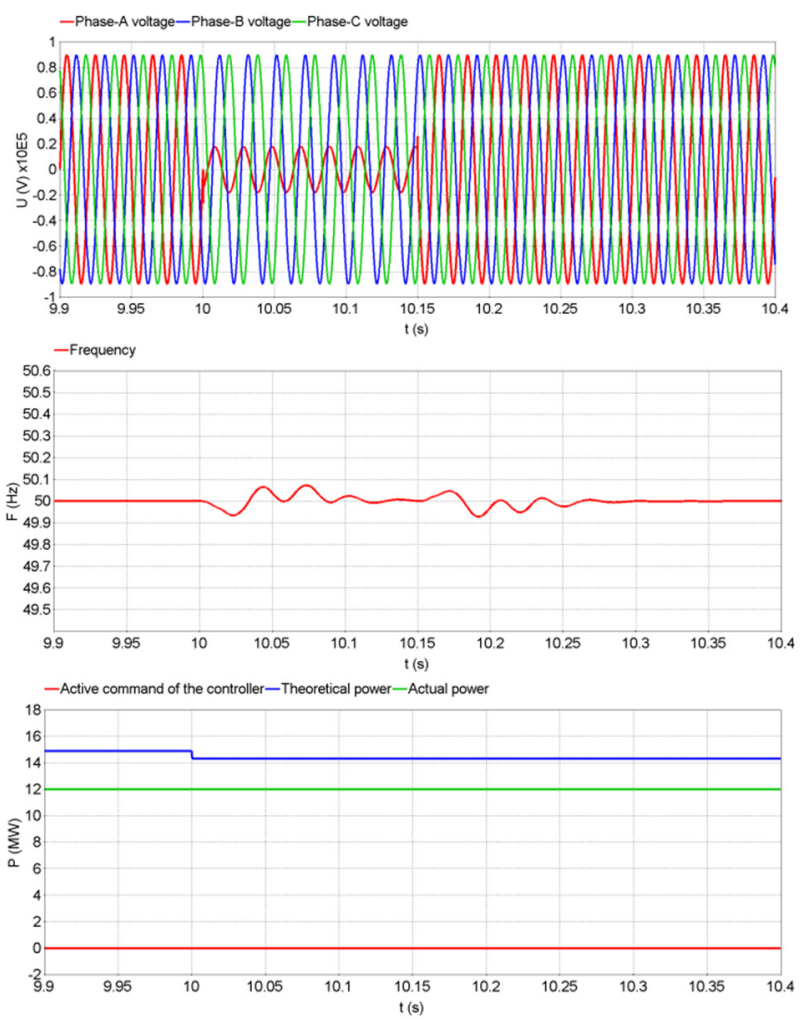

Fig.5 Response when phase-A voltage fell to $20 \%$ Un and the active command of AGC was $12 \mathrm{MW}$ at limited power under the working condition 1

Fig.6 is the waveform diagram when the phase-A, phase-B and phase-C voltage amplitude of the wind power station instantly stepped to $115 \%$ and two phase shifts of $60^{\circ}$ were made when the voltage stepped and restored. The figures show that during the high voltage traversing in the stimulated power grid, the active command of the FFR controller of the station did not act, that is, the FFR capacity of the station did not act reliably, meeting the requirement.

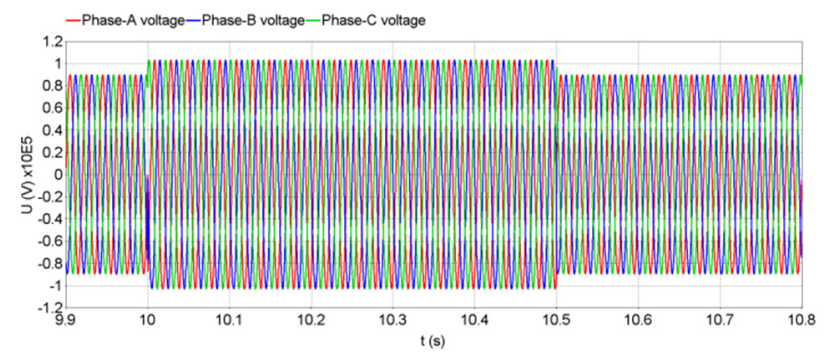



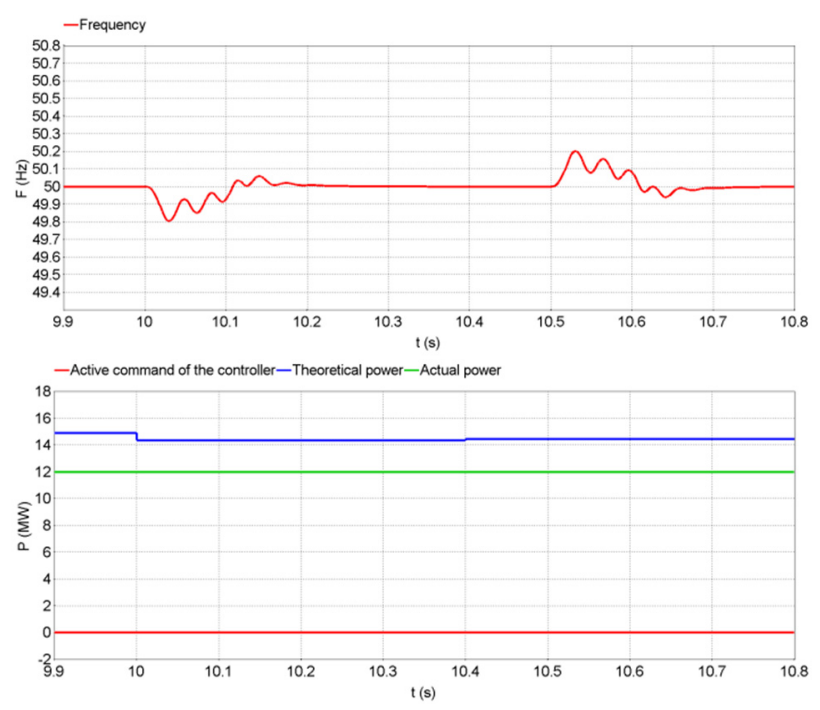

Fig.6 Response when phase-A, phase-B and phase-C voltage rose to $115 \%$ Un and the active command of AGC was $12 \mathrm{MW}$ at limited power under the working condition 1

\subsubsection{Result of the AGC coordination test}

Scopeview waveform analysis of RT-LAB was used to analyze the AGC coordination test. Fig.7 is the response to the commands of frequency stepping from $50 \mathrm{~Hz}$ to $50.20 \mathrm{~Hz}+10 \%$ increase in the rated capacity at secondary frequency regulation. The figure shows that after highfrequency step disturbance, the active command of the FFR controller of the station acted immediately to reduce active output and the AGC sent the increase command (reversely), while the actual power did not act and was properly locked.
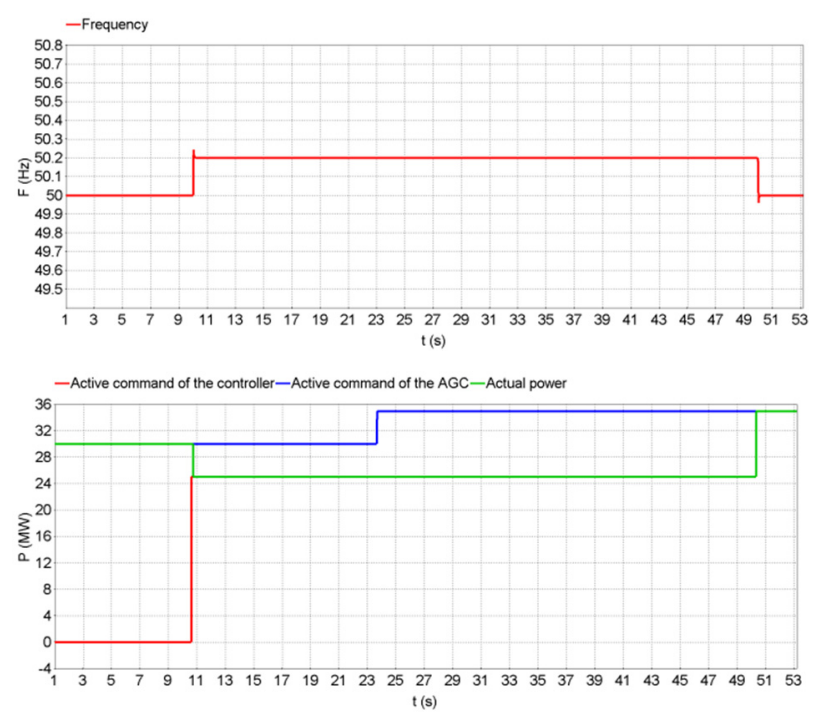

Fig.7 Response to the commands of frequency stepping from $50 \mathrm{~Hz}$ to $50.20 \mathrm{~Hz}+10 \%$ increase in the rated capacity at secondary frequency regulation under the working condition 3 (when the active command of the AGC fell from $30 \mathrm{MW}$ to 34.95 MW at limited power)

\section{Conclusion}

This study, based on the test method that had guided the test of the FFR capacity of new energy power stations for connection to the state power grid in Northwest China, investigated the hardware-in-the-loop simulation test method for FFR controllers of new energy power stations:

1) It made clear the hardware connection, modeling, working conditions of test and relevant parameters before the simulation test for authenticity and feasibility of the test.

2) In this study, the frequency step distance test, antidisturbance performance verification test and AGC coordination test were conducted for a new energy power station and introduced the response time, active power before and after step disturbance, active power of AGC, theoretical power and actual power were introduced. Besides, Scopeview waveform analysis of RT-LAB was used to analyze comprehensively the FFR controller of the new energy power station.

\section{Acknowledgments}

This project was supported partially by science and technology project of State Grid Shandong Electric Power Company, named Research on key technologies of data mining and security protection for supporting and Iegulating dloud plaform - topic 4: state asssment and deepening application of new energy data (No.520626190011).

\section{References}

1. TIAN Rubing, YANG Yupeng, LIU ZHiwu, MA Xiaodong. Research on primary frequency control for power system with participation of wind turbines. Heilongjiang Electric Power, 37(1): 42-53, (2015).

2. Zhou Tianpei, Sun Wei. Study on virtual inertia control for DFIG based wind farms with high penetration [J]. Proceedings of the CSEE, 37(2): 486495, (2017).

3. ZHANG Deqiang. Testing Analysis to Reactive Modulability of Large Scale Wind Farm. Inner Mongolia Electric Power, 29(6): 24-27, (2011).

4. QIN Rui, LIANG Fubo, ZHI Yong, BAI Runqing. Resarch on Reactive Power and Voltage Control Technology Based on Inverter in Photovoltaic Power Plants. Power Electronics, 50(3): 45-48, (2016).

5. MA Xiaowei, XU Haichao, LIU Xin, WANG Zhiwei, CHU Yunlong, LIU Xuchen, GUO Chongyang, BAO Sijia. A Test Method for Fast Frequency Response Function of Renewable Energy Stations in Northwest Power Grid. Power System Technology, 44(4): 13841391,(2020).

6. ZHANG Xiaolin, WU Zongguang, WANG Weixing, QIN Xiaodi. Design and Implementation of the Semiphysical Simulation for High-power Grid-connected Inverter. Power Electronics, 52(9): 104-111, (2018).

7. SHI Wei. Based on the simulation of low voltage 
crossing of the semi-physical test bench of the wind turbine. Shenyang University of Technology, (2013).

8. LIU Huil, GE Jun, GONG Yu1, LIU Hanmin, XIAO Fei. Optimization Scheme Selection of Wind Farm Participation in Grid Primary Frequency Modulation and Study of Wind-storage Coordination Control Strategy. Journal of Global Energy Interconnection, 2(1): 44-52,(2019).

9. Sun Xiaoqiang, Liu Xin, Cheng Lin, et al. Parameter setting of rapid frequency response of renewable energy sources in northwest power grid. Power System Technology, 43(5): 1760-1765, (2019).

10. Li Mingjie. Characteristic analysis and operational control of largescale hybrid UHV AC/DC power grids. Power System Technology, 40(4): 985-999, (2016).

11. YE Jing, LIN Tao, ZHANG Lei. Isolated grid unit commitment with dynamic frequency constraint considering photovoltaic power plants participating in frequency regulation. Transactions of China Electrotechnical Society, 32(13): 194-202, (2017).

12. LI Jianlin, MA Huimeng, YUAN Xiaodong. Overview on key applied technologies of arge-scale distributed Energy storage. Power System Technology, 41(10): 3365-3375, (2017). 